\title{
Stigma from medication use: an under recognised burden of care
}

Children with respiratory diseases take treatments for the self-management of symptoms and to maintain disease control. Often, these treatments need to be taken in social environments like school. Respiratory treatments can foster a feeling of difference and stigmatisation, which negatively impact on the quality of life and adherence to treatment. Such perceptions can lead to a less than optimal disease control, a vicious cycle leading to further social exclusion and stigma. This aspect of "burden of treatment" is poorly recognised by clinicians. Recognition of how treatments and clinical practice can contribute to stigma, can help address this burden of care.

\section{Educational aims}

- To understand the meaning of the term "stigma" within the context of respiratory health conditions and how medication or treatments can contribute to stigma in children and young people.

- To understand the potential impact of stigma on adherence, disease control and quality of life.

- To consider strategies to manage the stigma associated with health treatments across spheres of influence.

\section{Introduction}

Humans have evolved to exist in social groups, and therefore, interpersonal relationships and dynamics are important within everyday life. As humans develop throughout childhood and adolescence, they become increasingly aware of their sociocultural context and social belonging.

Stigma is typically a social process, experienced or anticipated, characterised by exclusion, rejection, blame or devaluation. These experiences are the result of perception or reasonable
Cite as: Kansra S, Calvert R, Jones S. Stigma from medication use: an under recognised burden of care. Breathe 2021; 17: 210002. 
anticipation of an adverse social judgment about a person or group [1].

Young people who have a chronic respiratory disease are often required to take medications to maintain their health status, with the administration of these medications often being required in public. Having to perform a behaviour that is objectively different from that of most of their peers can set an individual as "different" leading to a perceived choice, to perform the health behaviour or conform to social norms. While adherence in younger children may depend on parental attitudes towards the treatment, in the adolescent group, parental support and responsibility decreases. The child becomes more independent with medication during adolesence and there is more emphasis on increasing self-management at this stage in life [2].

As adolescents take on more responsibility for their self-management, psychosocial inferences from their peers may become more important, with adolescence being characterised by an increased need for social approval and peer acceptance as well as associated increases in risk-taking behaviour [3] The developmental awareness and the need for increased peer connection can come with a desire to minimise the difference, which would have possible implications for medication adherence.

Medication associated social burden is recognised as an important theme underlying medication-related behaviour [4]. This aspect of "burden of treatment" is poorly recognised by clinicians.

This article aims to contextualise the roots of difficulties inherent in "difference" associated with treatment for respiratory conditions, as well as some of the consequences and some suggestions to reduce the impact of the burden of care on an individual's life.

\section{Search strategy}

Relevant articles were identified by electronic searches of MEDLINE (from 1946 to present), EMBASE (from 1974 to present), EMCARE (from 1995 to present) and PsychINFO (from 1806 to present) using a combination of Medical subject headings and free text searches for "lung disorders", "bronchial disorders", "chronic respiratory tract disease", "long term ventilation", "cystic fibrosis", "asthma", "social stigma", "social acceptance or discrimination". Only manuscripts published in English were included. Articles were chosen according to their relevance for this review, and their bibliographies were also searched for references. Articles from our personal archives were also included.

\section{What is stigma?}

The most often quoted definition of stigma is "as an attribute that is deeply discrediting" and reduces the bearer "from a whole and usual person to a tainted discounted one" [5]. Following on from this definition, LINK and PHELAN [6] attempted to define stigma using a singular conceptualisation, which can be used as a foundation for the understanding of stigma within this article. This conceptualisation was arrived at having commented upon the varied and often differing descriptions that had previously existed in the literature. A breakdown of the criterion put forwards by LINK and PHELAN [6] can be found in table 1.

The natural human process of labelling discussed in relation to stigma is interpersonal in nature and occurs between an individual and

Table 1 Components of stigma

Component name
Distinguishing and labelling
difference
Associating human difference
with negative attributes
Separating "us" from "them"

Separating "us" from "them"

Status loss and discrimination

The dependence upon power

Information from [6].

\section{Description}

The oversimplification and categorisation of groups based on attributes is an attempt to make sense of our complex world.

Such categorisation leads to "taken for granted" labels that are applied in relation from one person to another.

Within a society specific labels become socially salient and therefore desirable.

Labelled differences can become linked to stereotypes, which can confer a socially undesirable characteristic about another person.

Those labelled with a socially undesirable characteristic can be viewed as fundamentally different from "us", which can lead to further generalised negative labelling due to a magnified perceived difference.

Due to being viewed as having socially undesirable characteristics a person may be set apart or devalued leading to a disadvantaged social position within a social hierarchy.

The exercise of power consciously or otherwise is often overlooked.

Being part of a socially desirable label allows choice and power over someone in a less powerful position with respect to things such as inclusion or opportunity. 
the expected social norms. It is also important to note that an individual can intra-personally ascribe these stigmatising labels to themselves. An individual may therefore perceive stigma in relation to their prior experience or in relation to possible or expected adverse social judgment in the future [7].

While the concept of stigma within social science literature has been around for more than half a century, there has recently been an increasing interest in health studies of stigma. Having a chronic medical condition, especially a condition which may need treatment in public, could be associated with all the component domains of stigma. WeIss et al. [1] arrived at the following formulation describing stigma in the context of clinical practice and healthcare policy:

"Stigma is typically a social process, experienced or anticipated, characterized by exclusion, rejection, blame or devaluation that results from experience, perception or reasonable anticipation of an adverse social judgment about a person or group. This judgment is based on an enduring feature of identity conferred by a health problem or health-related condition, and the judgment is in some essential way medically unwarranted. In addition to its application to persons or a group, the discriminatory social judgment may also be applied to the disease or designated health problem itself with repercussions in social and health policy."

Based on reviews of previous research ROSE et al. [8] have suggested that shame, blame and guilt may all fall within "stigma related experiences" for individuals who have been diagnosed with non-communicable respiratory diseases. Such experiences may lead to a delay in seeking medical help, diagnosis concealment, increased symptom severity and poor treatment adherence.

\section{Stigma and respiratory diseases}

Respiratory conditions bring about markers of difference in some people with respect to persistent coughs, being unable to engage in certain activities or having to take treatments such as inhalers. Research suggests that symptoms such as persistent coughing can "raise the spectre of contagion" leading to avoidance or concern, and can be disruptive to typical social interaction [9]. Visible differences such as coughing or needing to take treatment can lead to individuals experiencing stigma, whilst hidden differences, such as the genetic nature of conditions such as cystic fibrosis (CF), may also contribute to internalised stigma [10]. Children diagnosed with CF can experience stigma related to an inherited disease, which presents challenges to their management. In addition, stigma related to an inherited disease may be experienced to a greater degree within different ethnic or cultural subgroups [11].

While stigma related to respiratory illness is historically thought to be due to the physical attributes of the disease like cough and expectoration, with improvements in treatments, it is also likely that the need to take treatments is itself a marker of exceptionalism. Treatments for children with chronic respiratory diseases need to be taken regularly and are likely to impact on a child's social participation in and out of school, which may lead to a feeling of exclusion and generate a sense of social stigma.

Children's respiratory problems can be subject to acute worsening, which needs selfmanagement. This is best exemplified by asthma, where children may have asthma attacks in any setting and are expected to recognise and manage these by themselves or with assistance from their carers and teachers. For example, during lessons in school a child might have to stand up and ask to be excused from a lesson or to be allowed to take their inhaler during the lesson drawing attention to them and setting themselves as different from their peers. Teachers often inadvertently support the separation by serving as gatekeepers to permit or deny access to treatment and decisions are made according to their own previous experiences of asthma [12].

Children with asthma in school can be made to sit out of physical activities and report limited access to medication during school. Young people have also expressed concerns about teasing when medicating in front of their peers [13]. Even in schools where policies allow children to self-carry inhalers, children report difficulties in using the inhaler spacer combination due to anxiety or due to stigma about their asthma and medication use [14]. The metered dose inhaler, a valuable tool in managing asthma, has become a symbol of distinctiveness in classrooms around the world.

Asthma UK research reported that children can feel embarrassed or anxious about taking inhalers in public [15]. They may squirt or inhale medication away from the view from their peers especially with devices requiring a spacer. The common media and public perception of asthma treatments often neglect the use of the spacer device. Where the child musters enough courage to be able to tell the teacher that they want their inhaler, they still may not feel able to take it properly due to the fear of looking different or mocking from peers.

Not only does the design of treatment mechanisms take away a person's choice in being able to disclose or not disclose their health condition, but they can also be a source of embarrassment. A few children in the asthma UK study expressed concern about the shape of the inhalers and therefore felt embarrassed about using them [15]. We have heard similar concerns 
in our clinical practice. Understandably, to avoid negative stigma children engage in behavioural consequences that include ignoring and skipping treatment.

Children who are dependent on long-term mechanical ventilation (LTV) can appear frail and appear physically different to their peers. The life-sustaining treatments like tracheostomies and ventilators, also set them apart as different with families and children who need LTV feeling socially excluded [16]. Not only do parents feel that the lives of their children are discredited, but caring for a disabled child or being a sibling of a disabled child can lead to societal rejection. There are various components of stigmatising behaviour that both families and parents of these children experience [16].

While it is important to consider the impact of stigma on patients and families, evidence also suggests that stigma reverberates throughout healthcare settings and within staff groups to influence policy and working practices [17]. These findings have an implication for how we administer treatment and clinical practice. Clinicians' experiences and beliefs associated with symptoms and treatments may influence their decision making. If the clinician were to unilaterally offer a specific treatment because they felt that it would be less stigmatising, or to offer a treatment without considering the potential stigmatising impact, then the clinician may be unwittingly engaging in a stigmatising behaviour in itself or making a judgment that balances stigma against clinical need without asking for the child's perception. Offering something because of clinician perceptions, without involving the patient and asking about their wishes directly can be problematic and may omit a part of the person's lived experience and perceptions of specific treatments, which can be a barrier to effective treatment [15].

Furthermore, stigma in respiratory disease is impacted by broader cultural influences. For example, there is a belief in some south-east Asian families that asthma in their child will reduce the likelihood of a successful marriage [18]. Additionally, in western media several film and TV programmes continue to depict children with asthma as "weak", overly anxious people needing to toughen up. CLARK [12] highlighted the 1985 film The Goonies in setting the cultural understanding of an asthma attack stemming from a stress response, and Hitch depicts the protagonist being able to use willpower to overcome asthma, tossing his inhaler aside as he goes to kiss the woman he has been pursuing.

\section{Impact of medication-related stigma on health outcomes}

Medication and treatments in social environments can foster a feeling of separateness and can lead to stigma and embarrassment, which affect adherence as well as ability to seek help. These experiences may lead to a delay in seeking medical help, diagnosis concealment, increased symptom severity and poor treatment adherence and reduced physical activity, which has further health implications [8]. These factors inevitably lead to a detrimental impact on disease control. Poor control of a chronic disease leads to a vicious cycle whereby the flare-ups impair social participation and lead to social exclusion.

Research suggests that respiratory conditionrelated stigma can motivate the individual to hide their health condition through non-adherence to necessary medications [19]. Participants spoke of "not feeling normal" and one was quoted as saying "You cannot exactly feel normal because you're sat there like a lunatic with this ... machine in your mouth". While some participants described positive responses from others upon disclosure of CF, some were met with very negative comments leading to embarrassment or feeling the need to isolate themselves when performing treatment. In addition to routine treatment, hospital appointments and inpatient stays were said to interrupt school attendance and social relationships and could exacerbate the feelings of difference and perceived stigma in young people diagnosed with CF [20].

Circumventing treatment is a way to avoid other people learning about the disease but also a means of normification, i.e. to forget, for a while, that the disease exists. In a study examining barriers and facilitators to asthma care in schools, students described allowing symptoms to worsen because they did not want to interrupt activities to manage their asthma. Some students also described feeling left out of activities where they could not fully participate and expressed a desire for more inclusive activities that are safe for those with asthma [21].

Children needing LTV, who have significant disabilities, do not want to come across as different. They alter selected activities to appear normal to their friends. CARNEVALE [16] quoted a girl, who was ventilated only at night, that did not want to sleep over at her friends' houses because she did not want them to see her ventilator.

Adherence to effective treatments like inhalers is notably difficult and adherence behaviours are influenced by patient perceptions. A literature review assessing the perceptions of asthma patients in relation to treatment found that one of the important themes in relation to medication adherence was social stigma, especially in the adolescent age group [22]. These patients were likely to feel uneasy using their inhaler in public spaces. Similarly, social comparison with healthy peers around the illness can lead to feelings of embarrassment about their respiratory condition, and the beliefs of teachers and other adults can also contribute to this embarrassment or social stigma. Embarrassment related to use of medication was more likely to be seen in patients who reported low adherence [22]. 
Inappropriate self-management with high use of reliever inhalers in asthma is a risky behaviour and seems to be more evident in those who report stigma-related experiences. In a study of young adults with asthma, stigma was common; respondents described "just wanting to be like the rest and not to have to stop and put this thing in my mouth", asthma was "so embarrassing" and inhalers were something "to hide in a bag". Young adults who were taking inappropriately high amounts of reliever inhaler were more likely to report anger, resentment and stigmatisation [23].

Stigma derived from using medication may be detrimental to physical, emotional and social well-being. Such concerns need to be factored in when choosing treatments for children who attend school. In a systematic review and meta-synthesis of qualitative studies exploring medicationrelated burden and patients' lived experience with medicines, social burden of disease was identified as an important subtheme influencing medicationrelated behaviour. The authors found that medicines use associated social burden, such as social isolation and social stigma, often altered patients' relationships with family and friends. The fear of social stigma associated with medicine use had a significant psychological burden on patients' lives
Table 2 Impact of medication-related stigma on disease and health outcomes

- Concealment leading to delay in presentations and treatment

- Poor adherence to treatment

- Inappropriate treatment (e.g. overreliance on reliever inhaler in asthma)

- Psychological impact

- Poor participation in social activities and further social exclusion

- Poor disease control and more symptoms

that may have triggered further isolation. Conversely, the benefit of social support (e.g. family members) in assisting self-care and proper medicines use was evident [4].

A systematic review looking at stigma-related experiences of adults and patient outcomes in noncommunicable respiratory diseases in adults found that poorer health status scores were significantly associated with increased perceived stigma in asthma patients, although no link was found to hospital admissions [8]. The study also found that perceived stigma was significantly associated with dyspnoea and cough symptoms. Ahmad et al. [24] reported that self-stigma and self-esteem is a predictor of poor asthma control.

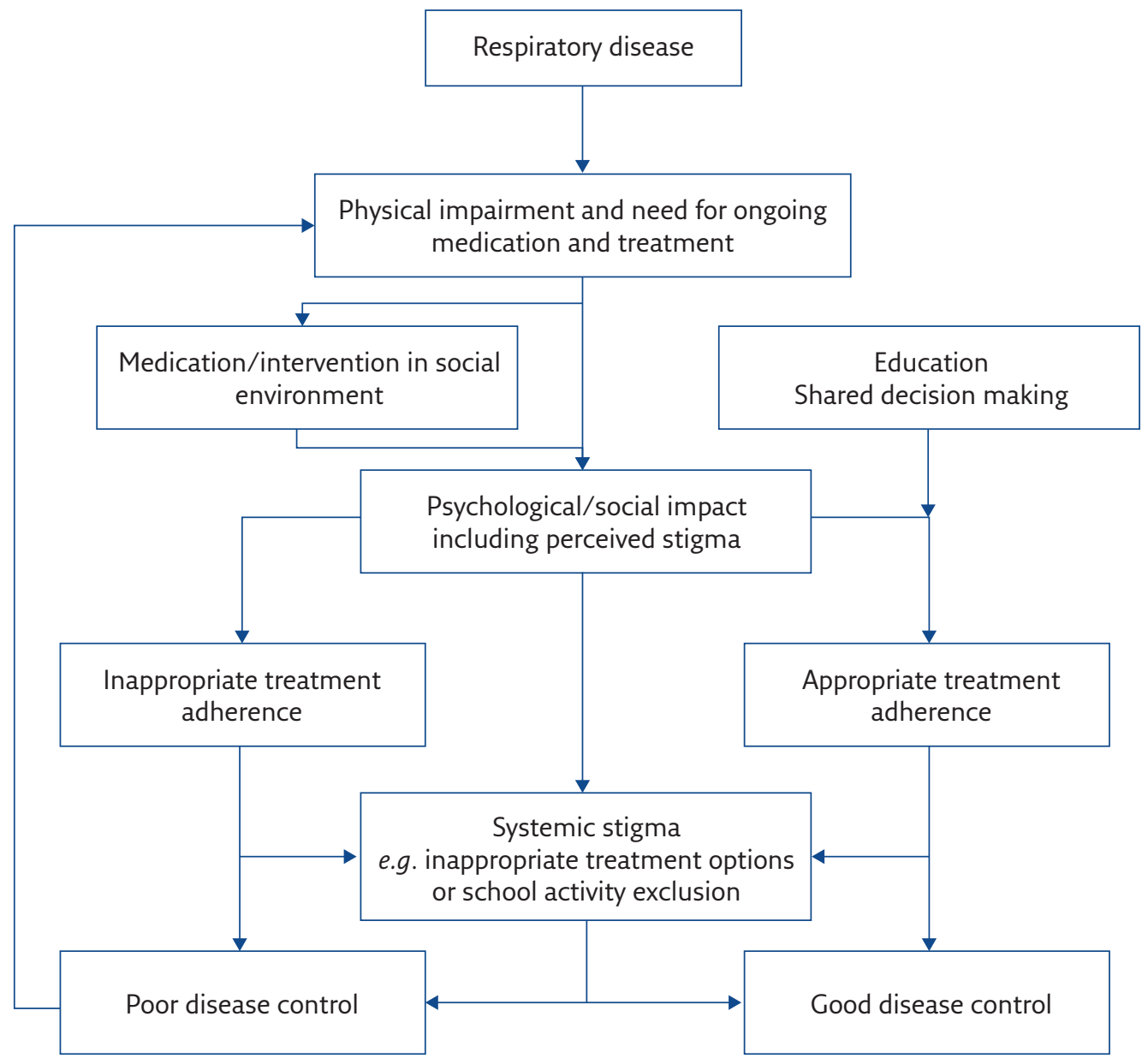

Figure 1 The interaction of respiratory disease, need for medication and stigma. 
Almomani et al. [25] looked at stigma in children with asthma and concluded that children who were less stigmatised had a better asthma control. The tool used to look at stigma was modified to ask questions about the embarrassment that asthma patients tend to feel about 1) telling people that they have asthma; and 2) using the inhalers in front of friends or relatives.

Poor disease control leads to a vicious cycle whereby the manifestations of the disease become more apparent and reinforce the stigmatisation. It is therefore paramount that the stigma associated with living with a respiratory condition and all the related experiences is fully explored when caring for these children and their families. The impact of medication-related stigma on disease and health outcomes is summarised in table 2.

Figure 1 is a diagrammatic representation of the interaction of respiratory disease, need for medication and stigma. The only thing that is static is the respiratory disease as everything else moves around the cycle. This is an ongoing process: just because someone is adhering well or has good disease control right now does not mean that they are not subject to psychological pressures/stigma or that this could change.

\section{Practical tips on how to manage the stigma associated with health treatments across all spheres of influence}

A detailed understanding of how health treatments can impact on perceived stigma and subsequent behaviours needs to take account of complex variables, including the individual's developmental

\section{Self-evaluation questions}

1. Which of the following can be considered stigma-related experiences?
a) Shame
b) Blame
c) Guilt
d) Embarrassment
e) All of the above

2. Stigma related to a respiratory condition and treatment leads to which of the following:
a) Poor disease control
b) Concealment of treatment
c) Poor adherence to treatment
d) Resilience
e) All of the above

3. Which of the following are examples of sociocultural influences which can impact health beliefs?
a) Inhalers are addictive
b) Children with asthma are weaker than their peers and should not exercise
c) Stock pictures of children taking inhalers without spacer
d) Change in diet can be a substitute for asthma treatments

4. The power dynamics between groups and individuals is important to consider in the perception of stigma. Which of the following are exercises of power (conscious or unconscious) that may influence someone's perception of stigma?
a) A teacher asking a young person not to engage in physical education (PE) due to concerns about their health
b) A group of children hiding another child's inhaler "for a joke"
c) A doctor prescribing a medication which requires a large medical device to administer without discussing it with the child
d) A child deciding they would rather play a video game instead of taking routine treatment
e) All of the above

5. Sophie is a 14-year-old with allergic asthma and eczema. Sophie has been provided a turbohaler by her asthma nurse as a single maintenance and reliever treatment (MART). Sophie has needed to take her inhaler on a few occasions in school and has been advised to sit out of PE lessons. Over the next few months Sophie's asthma becomes hard to control and her school attendance drops, and her mother has noticed a change in Sophie's mood. Sophie may benefit from which of the following interventions:
a) Educating teachers about her condition and agreeing a self-management plan in liaison with school
b) Once daily inhaler treatment
c) Discussion about various types of treatments available for her asthma and agreeing an inhaler which is acceptable and efficacious
d) Minimise school disruption by using technology to conduct reviews 
stage, the numerous personal, social and situational characteristics, and broader cultural influences. There are, however, some suggested strategies for addressing the stigma associated with respiratory treatments.

\section{For healthcare settings}

- Clinicians should continue to be mindful of the potential aversive feelings of embarrassment and social stigma associated with a young person's physical health and treatment and sensitively explore these during consultations.

- Clinicians are encouraged to reflect on their own inherent assumptions and biases that may influence their decision making in relation to the patient's sociocultural influences. The emphasis should be on shared decision making and individualisation.

- Clinicians are encouraged to attend to the power dynamics within consultations with young people to facilitate a helping relationship, wherein uncomfortable feelings such as embarrassment and shame can be talked about.

- Normalise and validate young people's experiences.

- Empower children and young people with education and knowledge about the disease and provide them with strategies for managing potential teasing/bullying associated with their health condition.

- Innovative ways of providing routine care of chronic respiratory conditions using technology may reduce hospital attendance and therefore absenteeism, thus minimising the difference between peers.

- Explore facilitating peer support structures for young people and their families.
- Consider referral for psychological intervention for shame-based difficulties if impacting on optimal physical health management.

\section{For educational settings}

- Support awareness and knowledge of respiratory diseases within educational settings to facilitate self-management with an agreed plan co-created by young people, their families, education and health professionals.

\section{Advocacy and community}

- Challenge common misconceptions portrayed through the media and provide more reliable, accurate representations as role models (e.g. Annie in This Is Us), and also include portraying accurate inhaler techniques to facilitate a societal change in attitude and reduce stigma associated with the disease and treatments.

- Concerted advocacy and education, especially targeted to communities where respiratory disease can be considered stigmatising.

The distinctly interpersonal experience of stigma can be directly related to having a respiratory condition, both with respect to diagnosis and treatment burden. Research suggests that the experience of stigma can have significant impact on how an individual interacts with their condition, especially in relation to treatment adherence and therefore their subsequent health.

Clinicians can help to address this by attending to the lived experience of their patients with a "stigma lens" in order to offer appropriate care and not add to the level of experienced stigma while maintaining appropriate health outcomes.

\section{Affiliations}

Sonal Kansra ${ }^{1}$, Rachel Calvert ${ }^{2}$, Steve Jones ${ }^{2}$

1Paediatric Respiratory Medicine, Sheffield Children's Hospital NHS Trust, Sheffield, UK. 'Paediatric Psychology, Sheffield Children's Hospital NHS Trust, Sheffield, UK.

\section{Conflict of interest}

S. Kansra has nothing to disclose. R. Calvert has nothing to disclose. S. Jones has nothing to disclose.

\section{References}

1. Weiss MG, Ramakrishna J, Somma D. Health-related stigma: rethinking concepts and interventions. Psychol Health Med 2006; 11: 277-287

2. Desai M, Oppenheimer JJ. Medication adherence in the asthmatic child and adolescent. Curr Allergy Asthma Rep 2011; 11: 454-464

3. Andrews JL, Foulkes L, Blakemore S-J. Peer influence in adolescence: public-health implications for COVID-19. Trends Cogn Sci 2020; 24: 585-587.

4. Mohammed MA, Moles RJ, Chen TF. Medication-related burden and patients' lived experience with medicine: a systematic review and metasynthesis of qualitative studies. BMJ Open 2016; 6: e010035.

5. Goffman E. Stigma: Notes on the Management of Spoiled Identity. London, Penguin Psychology, 1990; p. 173.

6. Link BG, Phelan JC. Conceptualizing stigma. Annu Rev Sociol 2001; 2: 363-385

7. Scambler G. Health-related stigma. Sociol Health IIIn 2009; 31: 441-455.

8. Rose S, Paul C, Boyes A, et al. Stigma-related experiences in non-communicable respiratory diseases: a systematic review. Chron Respir Dis 2017; 14: 199-216. 


\section{Suggested answers}

1. e.

2. $a, b, c$.

3. $a, b, c, d$.

4. a, c.

5. a, c, d
9. Berger BE, Kapella MC, Larson JL. The experience of stigma in chronic obstructive pulmonary disease. West J Nurs Res 2011; 33: 916-932.

10. Pakhale S, Armstrong M, Holly C, et al. Assessment of stigma in patients with cystic fibrosis. BMC Pulm Med 2014; $14: 76$.

11. Chakraborty S, Krishnanathan T, Nwokora C, et al. Ethnic diversity in CF: facing the complexities. Pediatr Pulmonol 2019; 54: Suppl., 406.

12. Clark CD. Asthma episodes: stigma, children, and Hollywood films: asthma, children, and Hollywood films. Med Anthropol Q 2012; 26: 92-115.

13. Walker TJ, Reznik M. In-school asthma management and physical activity: children's perspectives. J Asthma 2014; 51: 808-813.

14. Sridharan G, Spalding A, Press VG, et al. Barriers and facilitators to self-carry of inhalers in school: a qualitative study of children with asthma. Am J Respir Crit Care Med 2017: 195: A3327.

15. De Simoni A, Horne R, Fleming L, et al. What do adolescents with asthma really think about adherence to inhalers? Insights from a qualitative analysis of a UK online forum. BMJ Open 2017; 7: e015245.

16. Carnevale FA. Revisiting Goffman's Stigma: the socia experience of families with children requiring mechanical ventilation at home. J Child Health Care 2007; 11: 7-18.

17. Nyblade L, Stockton MA, Giger K, et al. Stigma in health facilities: why it matters and how we can change it. $B M C$ Med 2019; 17: 25.

18. Lakhanpaul M, Culley L, Robertson N, et al. A qualitative study to identify parents' perceptions of and barriers to asthma management in children from South Asian and White British families. BMC Pulm Med 2017; 17: 126.

19. Jones S, Babiker N, Gardner E, et al. Promoting adherence to nebulized therapy in cystic fibrosis: poster development and a qualitative exploration of adherence. Patient Prefer Adherence 2015; 2015: 1109-1120.

20. D'Auria JP, Christian BJ, Henderson ZG, et al. The company they keep: the influence of peer relationships on adjustment to cystic fibrosis during adolescence. J Pediatr Nurs 2000; 15 175-182.

21. Naman J, Press VG, Vaughn D, et al. Student perspectives on asthma management in schools: a mixed-methods study examining experiences, facilitators, and barriers to care. $J$ Asthma 2019; 56: 1294-1305.

22. Lycett $\mathrm{H}$, Wildman $\mathrm{E}$, Raebel EM, et al. Treatment perceptions in patients with asthma: synthesis of factors influencing adherence. Respir Med 2018; 141: 180-189.

23. Cole $S$, Seale $C$, Griffiths $C$. 'The blue one takes a battering' why do young adults with asthma overuse bronchodilator inhalers? A qualitative study. BMJ Open 2013; 3 e002247.

24. Ahmad S, Ismail Al, Zim MAM, et al. Assessment of self-stigma, self-esteem, and asthma control: a preliminary cross-sectional study among adult asthmatic patients in Selangor, Malaysia. Front Public Health 2020; 7: 420.

25. Almomani BA, Al-Qawasmeh BS, Al-Shatnawi SF, et al. Predictors of proper inhaler technique and asthma control in pediatric patients with asthma. Pediatr Pulmonol 2021: 56 : 866-874. 